\title{
NUEVA TÉCNICA DE REPARACIÓN DE LOS DEFECTOS DEL COMPARTIMENTO ANTERIOR MEDIANTE IMPLANTE SINTÉTICO Y CUBIERTA BIOLÓGICA: ABORDAJE, FIJACIÓN Y ANCLAJE TRANSOBTURADOR.
}

\author{
Jesús Moreno Sierra, Sara B. Prieto Nogal, Mํ. Isabel Galante Romo, Luis E. Resel Folkersma y \\ Angel Silmi Moyano.
}

Cátedra y Servicio de Urología Hospital Clínico San Carlos. Universidad Complutense. Madrid. España.

\begin{abstract}
Resumen.- INTRODUCCIÓN: El sistema transobturatriz para la corrección de los prolapsos de la pared anterior de la vagina ejemplifica la tendencia actual de la cirugía del suelo pélvico. Puede ser considerado como una vía de abordaje y como un sistema de fijación de la malla, de forma contrapuesta a las mallas utilizadas para la corrección del cistocele que pueden dejarse libres en un intento de corregir el prolapso mediante la creación de un tejido fibrótico secundario a la colocación de la malla (biológica o sintética).
\end{abstract}

OBJETIVO: Describir los elementos que componen el sistema Avaulta anterior, sus indicaciones y la técnica quirúrgica mediante la que se implanta y ajusta adecuadamente.
MÉTODOS: El método quirúrgico consta de cinco pasos: 1ㅇ incisión vaginal media y disección de la mucosa vaginal, 2e) Identificación del agujero obturador, diseño y realización de dos mini-incisiones superiores en ambos pliegues genitofemorales y otras dos ubicadas a $3 \mathrm{~cm}$ por debajo y a $7-2 \mathrm{cms}$ laterales a las primeras, 3ㅇ Introducción y paso de las agujas, pasando por la parte superior del agujero obturador, paralelas a la rama isquiopubiana y una vez pasada se enhebra la aguja con el "brazo" del implante, 4의 Introducción y paso de las agujas por las mini-incisiones inferiores, pasando por la parte inferior del agujero obturador en sentido vertical y dirigiendo la aguja con control bimanual hacia la teórica localización del cuello uterino, con conexión y enhebrado del brazo inferior de la malla sobre la aguja de punción y 5ol Ajuste sin tensión de la malla y cierre de las incisiones vaginal e inguinales.

CONCLUSIONES: 1. Se trata de una técnica reproducible que permite corregir adecuadamente los defectos del compartimento anterior vaginal. 2. El diseño y tecnología de Avaulta pretende corregir los defectos del compartimento anterior, basándose en los principios de la malla ideal.

Palabras clave: Compartimento anterior. Reparación cistocele. Malla biológica. TOT.

Summary.- Transobturator systems for anterior vaginal wall prolapse repair exemplify the current trend in pelvic floor surgery. They may be considered an approach and also a mesh fixation system, in opposition to free mesh cystocele repair where they work by the creation of fibrotic tissue after mesh implant (biological or synthetic). 
OBJECTIVES: To describe the elements of the Avaulta anterior system, its indications and the surgical technique to implant it and adequately adjust it.

METHODS: The operation has five steps: 11 midline vaginal incision and mucosal dissection, 2) Obturator foramen identification, design and performance of 2 superior mini incisions in both the genitofemoral folds, and another two $3 \mathrm{~cm}$ below and $1-2 \mathrm{~cm}$ lateral to them, 3) Needle introduction and passage through the upper portion of the obturator foramen, parallel to the ischiopubic ramus, and once past needle charging with the arm of the implant, 4) Needle introduction and passage from the inferior incisions vertically through the inferior portion of the obturator foramen, directing the needle with bimanual control to the theoretical localization of the uterine cervix, with connection and charging of the inferior arm of the mesh. 5) Tension free adjustment of the mesh and closure of the incisions.

CONCLUSIONS: 1. It is a reproducible technique that adequately corrects the anterior vaginal compartment defects. 2. The design and technology of Avaulta aims to correct the anterior compartment defects, based on the principles of ideal mesh.

Keywords: Anterior compartment. Cystocele repair. Biological mesh. TOT.

\section{INTRODUCCIÓN}

Se define el cistocele como el descenso vesical, prolapsado a través de la vagina que se produce como consecuencia de alteraciones en el músculo aponeurótico y en los ligamentos del suelo pélvico $(1,2)$. En la nomenclatura actual se engloba como un defecto del compartimento anterior. El principal soporte muscular de los órganos pelvianos son los músculos coccígeo y elevador del ano, y dentro de este el papel primordial lo desempeña su aponeurosis, con cuatro condensaciones que forman los ligamentos del suelo pélvico: Pubouretrales, Uretropélvicos, Pubocervicales y Cardinales-Sacrouterinos (1-5).

La aparición de los diferentes grados de cistocele viene determinada por la combinación de defectos de los elementos antes mencionados, y se dividen en: Defecto central (el prolapso vesical se produce en la línea media por defecto del ligamento pubocervical), Defecto lateral (descenso de la vejiga por debilidad o desprendimiento de la inserción los ligamentos pubocervical y uretropelviano en la pared lateral pélvica) y Defecto combinado (el más frecuente y se produce por la asociación de los dos anteriores, dando lugar a los grandes cistoceles) $(1,2,5)$.
El campo del uso de mallas para la corrección del cistocele es un campo emergente donde van apareciendo múltiples técnicas nuevas. Los estudios realizados aportan resultados esperanzadores. La malla ideal debe ser biocompatible, inerte, hipoalergénica, no inflamatoria, estéril, no carcinogénica, resistente al estrés mecánico y disponible en un formato cómodo para su uso clínico (6).

El sistema transobturatriz para la corrección de los prolapsos de la pared anterior de la vagina ejemplifica esta tendencia de las nuevas técnicas quirúrgicas. Puede ser considerado como una vía de abordaje y como una fijación de la malla, de forma contrapuesta a las mallas utilizadas para la corrección del cistocele que pueden dejarse libres en un intento de corregir el prolapso mediante la creación de un tejido fibrótico secundario a la colocación de la malla (biológica o sintética) (6-10). El diseño y tecnología de Avaulta pretende corregir los defectos del compartimento anterior, basandose en los principios de la malla ideal.

\section{OBJETIVO}

Describir los elementos que componen el sistema Avaulta anterior, sus indicaciones y la técnica quirúrgica mediante la que se implanta y ajusta adecuadamente.

\section{MATERIAL Y MÉTODO}

\section{Indicaciones}

Esta malla está indicada en pacientes sintomáticas con defectos moderados y severos del compartimento anterior (cistocele) con o sin prolapsos de cuello uterino asociado. Por otra parte puede asociarse a otras técnicas correctoras del suelo pélvico (prolapso de cúpula, enterocele y/o rectocele). En grandes prolapsos de utero puede asociarse a histerectomía vaginal, con la finalidad de realizar una restitución del suelo pélvico, lo más fisiológica posible.

\section{Protocolo de estudio}

El diagnóstico se realiza con una adecuada historia y exploración física y se completa con la realización de las siguientes pruebas complementarias: uretrocistografía y estudio urodinámico, ecografía urológica y opcionalmente uretrocistoscopia, ecografía ginecológica y resonancia magnética del suelo pélvico.

Previo a la intervención quirúrgica el paciente deberá ser informado sobre las características del 


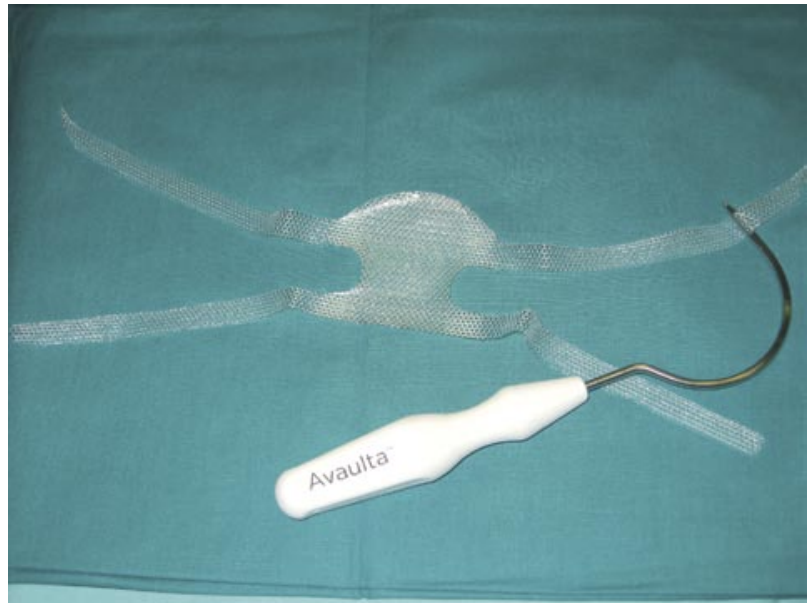

FIGURA 1. Implante anterior y aguja de punción.

procedimiento y el sistema a implantar, con sus ventajas y posibles inconvenientes, riesgos y alternativas a este sistema. Este consentimiento que inicialmente será verbal, deberá ser firmado por la propia paciente.

\section{Características de la malla implantable "Avaulta an- terior"}

Es una prótesis mixta (implante fabricado con polipropileno protegido con colágeno porcino), compuesta por una malla para la corrección del defecto central, cuatro prolongaciones o brazos laterales autofijables para la corrección del defecto lateral por vía transobturatriz (Figura 1) y un sistema de agujas que nos permitan realizar la punción del agujero obturador con la finalidad de extraer las prolongaciones de la malla e inducir su fijación y autoanclaje.

\section{Material necesario}

1. Instrumental general: pinzas, bisturí, tijeras, pinzas de disección, pinzas de mosquitos, separador y valvas vaginales

2. Instrumental específico: aguja de punción para abordaje transobturatorio e implante anterior (malla de polipropileno protegido con colágeno)

\section{Técnica}

\section{Anestesia}

El procedimiento puede realizarse bajo anestesia raquídea o general.

\section{Preparación del paciente.}

Limpieza mecánica de la vagina y recto (opcionalmente), profilaxis antibiótica, higiene y aseptización del área hipogástrica y urogenital con povidona yodada, sondaje uretrovesical (Foley $n^{\circ} 18$ ), colocación del paciente en posición adecuada (litotomía y moderado Trendelemburgl y colocación del campo quirúrgico.

\section{Técnica quirúrgica paso a paso.}

La técnica quirúrgica consta de cinco pasos o etapas:

\section{Primer paso}

Este paso precisa de una adecuada colocación de la paciente en posición uroginecológica y posterior hidrodisección (Figura 2), incisión vaginal media y disección de la mucosa vaginal en sentido lateral hacia el agujero obturador.

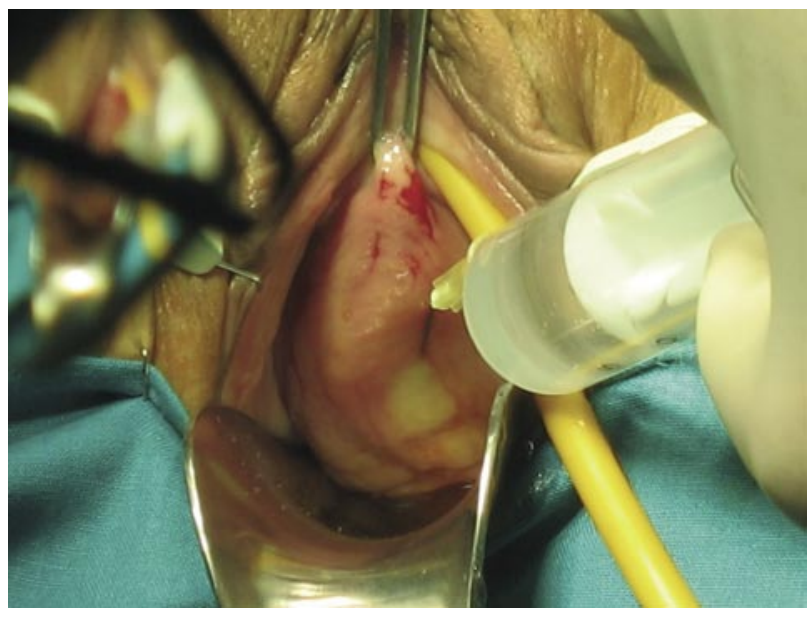

FIGURA 2. Hidrodisección.

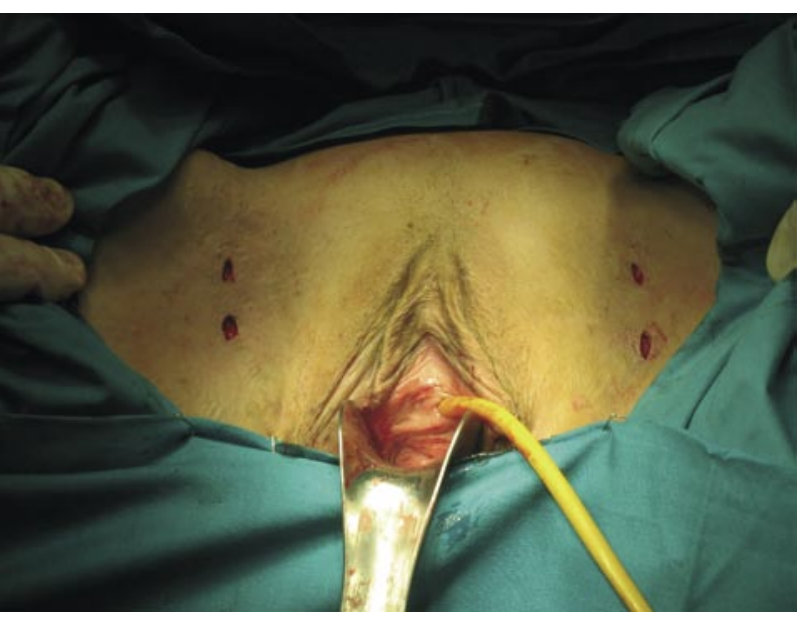

FIGURA 3. Diseño de las incisiones. 

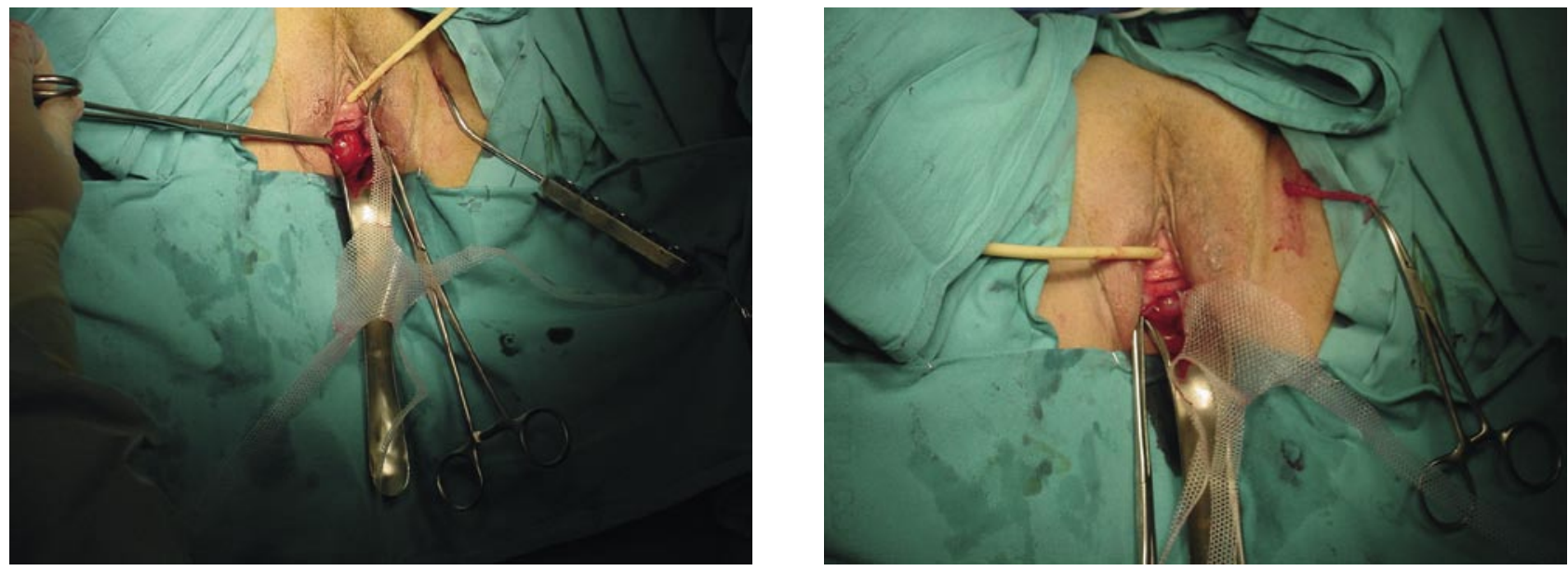

FIGURA 4.

\section{Segundo paso}

Identificación del agujero obturador. Diseño y realización de dos mini-incisiones superiores (de aproximadamente 1-1'5 cms) en ambos pliegues genitofemorales y otras dos ubicadas a $3 \mathrm{~cm}$ por debajo y a $1-2 \mathrm{cms}$ laterales a las primeras (también de aproximadamente 1-1'5 cms) (Figura 3).

\section{Tercer paso}

Introducción y paso de las agujas por las mini-incisiones superiores (a nivel del clítoris), pasando por la parte superior del agujero obturador, paralelas a la rama isquiopubiana (Figura 4). Control bimanual del paso de la aguja de punción y una vez pasada y enhebrada la aguja con el "brazo" del implante (Figura 5), se repite el procedimiento en el lado contralateral.

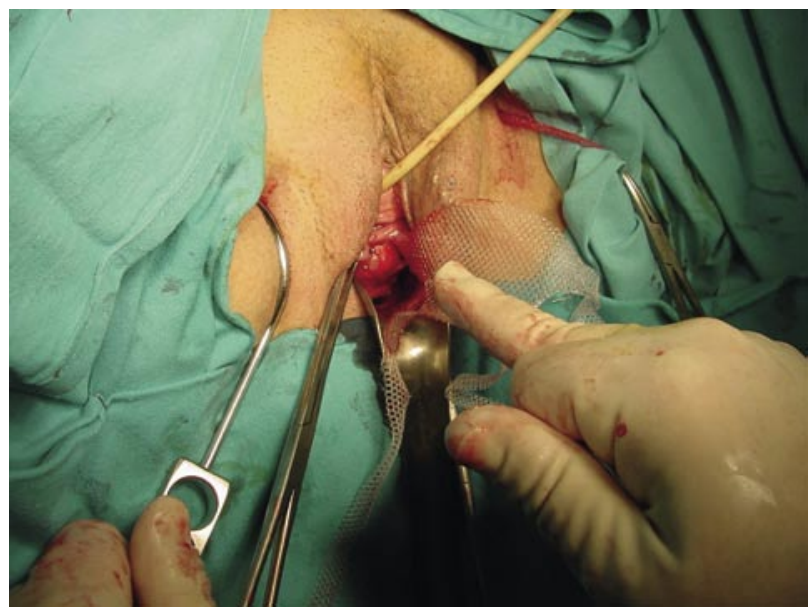

\section{Cuarto paso}

Introducción y paso de las agujas por las mini-incisiones inferiores $(a) 3 \mathrm{cms}$ por debajo y 1 $2 \mathrm{cms}$ laterales a las localizadas a nivel superior), pasando por la parte inferior del agujero obturador en sentido vertical y dirigiendo la aguja con control bimanual hacia la teórica localización del cuello uterino, con conexión y enhebrado del brazo inferior de la malla sobre la aguja de punción. Se repite el mismo procedimiento en el lado contralateral.

\section{Quinto paso}

Ajuste sin tensión de la malla (Figuras 6 y 7 ). Opcionalmente y sobre todo en los casos de prolapso cervico-uterino, puede fijarse la porción inferior del cuerpo de la malla a los laterales del cervix (Figura 8). Esta maniobra nos permitirá en muchas ocasiones

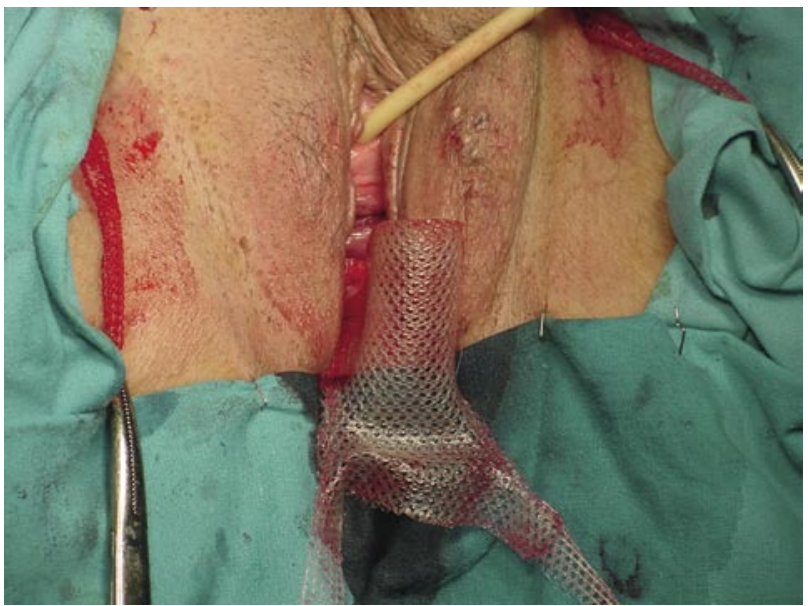




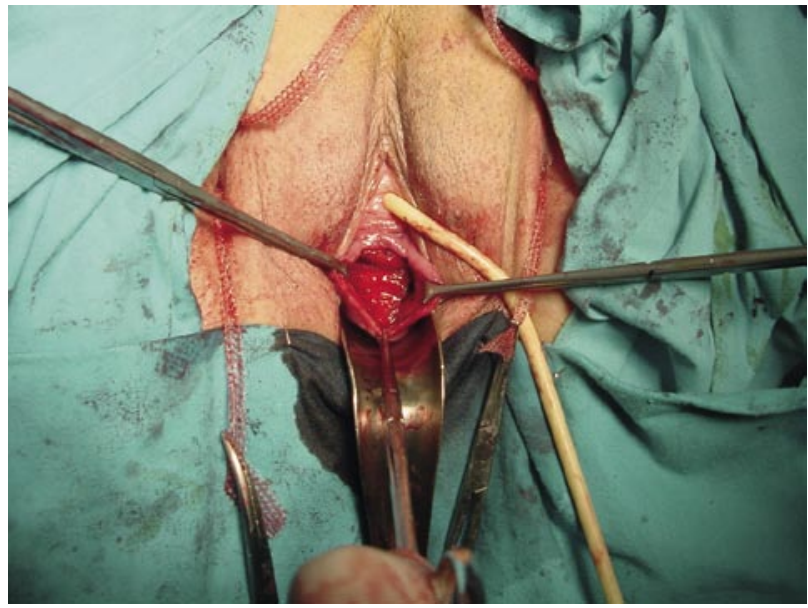

FIGURA 6.

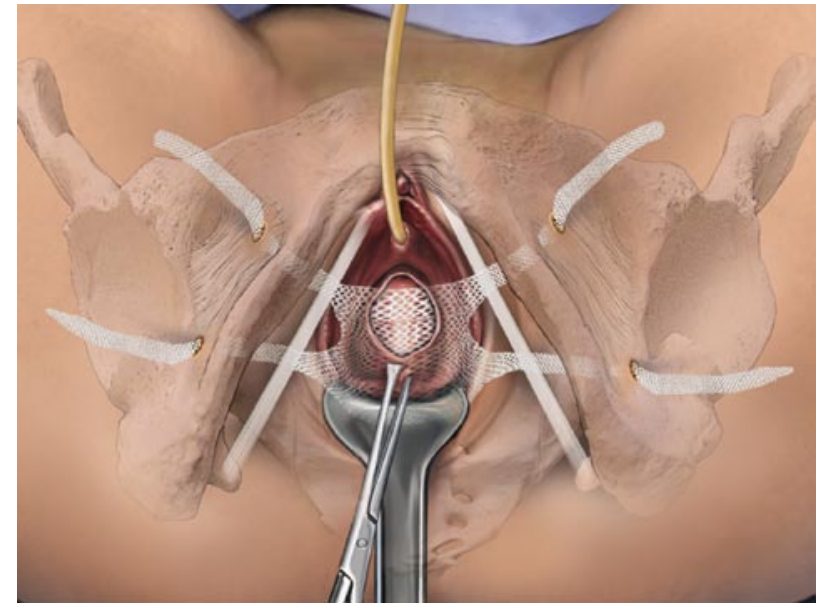

FIGURA 7. corregir este tipo de prolapso asociado y evitar la amputacion de cuello uterino o incluso la histerectomía. Cierre de las incisiones vaginal e inguinales.

Se finalizará comprobando el estado de continencia de la paciente y colocación de un malla suburetral TOT en los casos de incontinencia manifiesta.

\section{DISCUSIÓN}

El concepto de "biocirugía" defiende la idea de que los cirujanos de este siglo deben dejar de lado la concepción netamente mecánica de las cirugías, ya que trabajan en tejidos vivos, que tienen sus

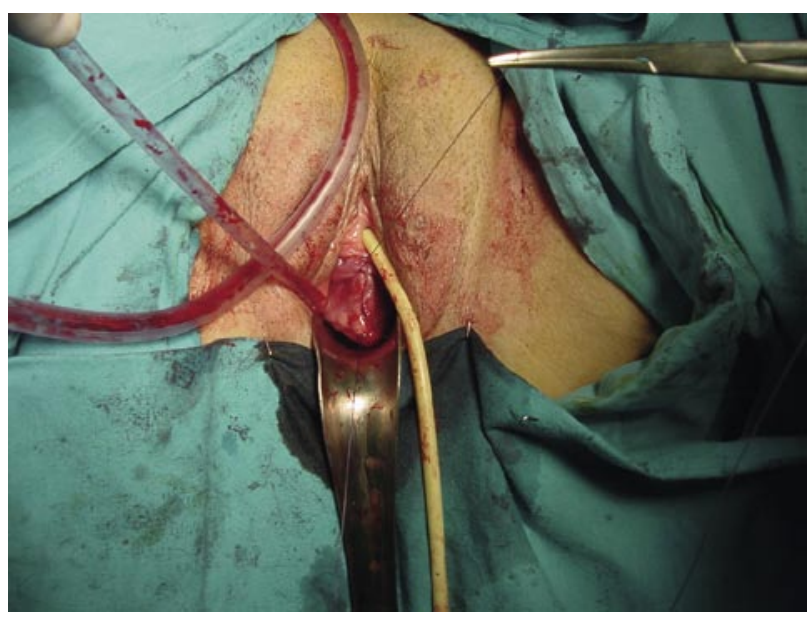

FIGURA 8. propias leyes de cicatrización y reparación. La biocirugía revaloriza los principios de la cirugía clásica asociándolos al refuerzo de los tejidos inducidos por biomateriales. Estos principios son: abordaje mínimamente invasivo, investigación de planos anatómicos y fisiológicos, mínimo trauma, respeto a la ingeniería de tejidos y sus ecosistemas específicos, aportar soluciones propias, que estimulan y canalizan reacciones en el organismo, donde son necesarias, ausencia de fijación (la sutura causa necrosis e infección) y resultados anatómicos y funcionales inmediatos. Por este motivo nos parece una idea muy atractiva corregir los defectos del compartimento anterior con este tipo de intervenciones y con este tipo de malla mixta.

Los resultados obtenidos inicialmente con esta novedosa técnica son buenos, en lo referente a corrección del prolapso, aunque con una tasa de recurrencia aproximada al $30 \%$ en el primer año. Hasta la fecha no han sido documentadas complicaciones reseñables ni efectos secundarios, si bien es cierto que no disponemos aún de experiencia a largo plazo. Por este motivo esperaremos un tiempo prudencial para evaluar correctamente su efectividad en nuestra serie.

De forma análoga a lo que ocurre cuando se deja una malla libre, pueden utilizarse materiales biológicos y sintéticos para la corrección del cistocele por abordaje transobturatríz, si bien es cierto que aún no se han comercializado prótesis biológicas de cuatro anclajes, que permitan corregir este tipo de prolapso. Por tanto, si queremos usarlas debemos construirlas en el propio quirófano, utilizando como base o patrón una malla biológica, que deberá ser recortada o realizar el diseño de un malla cuya base sea biológica y los anclajes de polipropileno. 
En cuanto a la colocación de una malla suburetral TOT para prevenir o corregir la incontinencia de esfuerzo existen dos alternativas o posturas: colocación en caso de incontinencia manifiesta y colocación de forma preventiva.

\section{CONCLUSIÓN}

- Se trata de un sistema reproducible que permite corregir adecuadamente los defectos del compartimento anterior vaginal.

- El diseño y tecnología de Avaulta pretende corregir los defectos del compartimento anterior, basandose en los principios de la malla ideal.

\section{BIBLIOGRAFÍA Y LECTURAS RECOMENDADAS (*lectura de interés $y$ ** lectura fundamental)}

**1. MORENO SIERRA, J.; DE LA PEÑA ZARZUELO, E. y RESEL ESTÉVEZ, L.: "Cirugía del cistocele, en Resel, L y Moreno, J: Atlas de Incontinencia Urinaria, $1^{\text {a }}$ edición. Madrid; 261267. 2000.

**2. BOCARDO, G.; MORENO, J.; REDONDO, E. y cols.: "Manejo de la incontinencia en pacientes con cistocele moderado y severo en: Resel, L; Sil- mi, A y Moreno, J: Incontinencia Urinaria”. Clin. Urol. Compl. 8; 459-472. 2000.

*3. SHULL, B.L.; BENN, T.J.: "Surgical management of prolapse of anterior vaginal segment: AN analysis of support defects, operative morbidity and anatomic outcome". Am. J. Obstect. Gynecol.; 171: 1429-39. 1994.

*4. RAZ. S., LITTLE, N.A.; JUMA, S.: "Female Urology". In: Walsh PC, Retik AB, Stamey TA, Vaughan DE, editors. Campbell's Urology. $6^{\mathrm{a}}$ ed. Philadelphia:.p.2782-828. 1992.

*5. SOLÉ- ROSELLÓ, A.; VILA, J.: "Incontinencia urinaria femenina". En: Jiménez Cruz JF, Rioja LA editores. Tratado de Urología. Tomo II. Barcelona: Ed. Prous;.p.1663-90. 1993.

**6. BEGLEY, J.S., KOBASHI, K.C.: "The use of graft materials in anterior compartment pelvic reconstruction". Curr. Urol. Rep. Oct; 5(5):397402. 2004.

**7. PALMA, P.; RANE, A.; RICCETTO, C. Y cols.: "Corrección transobturatriz de los cistoceles". Actas Urol. Esp. Jan; 29:89. 2005.

*8. DEVAL, B.; HAAB, F.: "What's new in prolapse surgery?". Curr. Opin. Uro., Volume 13.July. 315323.2003

**9. MAHER, C.; BAESSLER, K.; GLAZENER, C.M.: "Surgical management of pelvic organ prolapse in women". Cochrane Database Syst Rev. Oct 18; CD004014. 2004.

*10. HERNÁN BRAUN, B. Y cols.: "Prolapso genital severo: Consideraciones clínicas, fisiopatológicas y de técnica quirúrgica al momento de su corrección”. Rev Chil Obstet Gineco; 69,149. 2004. 